\title{
Oralidad, lectura y escritura competencias mediadoras del aprendizaje del currículo de Química: el caso del equilibrio químico
}

\author{
Orality, reading and writing mediator competences of the learning of the chemistry \\ curriculum: the case of the chemical equilibrium
}

\section{Oralidade, leitura e escrita. Competências mediadoras da aprendizagem do currículo de química: o caso do equilíbrio químico}

\author{
Boris Fernando Candela1 ${ }^{1}$
}

Recibido: abril de 2019

Aceptado: noviembre de 2019

Para citar este artículo: Candela, B.F. (2020). Oralidad, lectura y escritura competencias mediadoras del aprendizaje del currículo de Química: el caso del equilibrio químico. Revista Científica, 37(1), 18-29. Doi: https://doi. org/10.14483/23448350.14839

\section{Resumen}

Los investigadores de la educación en Química han considerado que una de las metas clave del currículo de esta disciplina es la alfabetización científica en el sentido fundamental (hablar, leer y escribir) y derivado (comprender los productos y proceso de la química). Si bien este propósito ha sido un elemento clave de las actuales reformas curriculares de esta disciplina, en la práctica docente ha resultado difícil de lograr; dado que los profesores se han centrado en asistir a los estudiantes en la comprensión de los modelos teóricos, descuidando el desarrollo de las competencias lingüísticas de la oralidad, la lectura y la escritura como elementos constitutivos de la Química. En esta investigación se aplicó una metodología cualitativa por estudio de casos, la cual requirió para la recolección de los datos de fuentes documentales como: vídeos, notas de campo, relatos narrativos y trabajos de los estudiantes. Los resultados permiten ver que para lograr la anhelada alfabetización en el aula de Química, se requiere que el profesor asista de manera consciente a sus estudiantes en el desarrollo integrado de las competencias lingüísticas de hablar, leer y escribir de y sobre la Química dentro de un contexto sociocultural. Palabras clave: aprendizaje, escritura, equilibrio químico, educación, lectura, oralidad.

\begin{abstract}
The researchers of the education in chemistry have considered that one of the key goals of the curriculum of this discipline, is the scientific literacy in the fundamental sense (to speak, to read and to write) and derivative (to understand the products and process of chemistry). Although, this purpose has been a key element of the current curricular reforms of this discipline, in the teaching practice it has been difficult to achieve; given that teachers have focused on assisting students in the understanding of theoretical models, neglecting the development of
\end{abstract}


linguistic competences of orality, reading and writing as constitutive elements of chemistry. A qualitative methodology was used for case studies, which required the collection of data from documentary sources such as: videos, field notes, narrative stories and student work. The results allowed us to see that to achieve the desired literacy in the chemistry classroom, the teacher is required to consciously assist his students in the integrated development of the speaking, reading and writing language skills of and about chemistry, within a sociocultural context.

Keywords: chemical balance, education, learning, orality, reading, writing.

\section{Resumo}

Os investigadores da educação em Química consideraram que um dos objetivos do currículo desta disciplina é a alfabetização científica no sentido fundamental (hablar, ler e escrever) e derivar (incluir os produtos e o processo da química). Se este propósito tiver sido um elemento básico das reformas curriculares desta disciplina, na prática docente terá resultado difícil de lograr; dado que os professores estão centrados em ajudar os alunos na compreensão dos modelos teóricos, descobrindo o desenvolvimento das competências linguísticas da oralidade, a aula e a escrita como elementos constitutivos da química. Nesta investigação, é aplicada uma metodologia qualificada pelo estúdio de casos, o requerido para a recuperação de dados de documentos documentais como: vídeos, notas de campo, relatos narrativos e trabalhos dos estudantes. Os resultados permitidos para lograr a alfabetização anhelada na aula de Química, se necessário, para o professor assistente de gestão consciente de seus alunos e para o projeto integrado de competências lingüísticas de hablar, ler e escrever sobre a química dentro de um contexto sociocultural.

Palavras-chaves: oralidade, lectura, escritura, aprendizagem, equilíbrio químico, educação.

\section{Introducción}

Las reformas en la educación en Ciencias han abordado diferentes aspectos de la enseñanza y aprendizaje de estas disciplinas. Sin embargo, estas se han centrado en el currículo junto a las metas, fines y materiales curriculares, descuidando otros elementos que desempeñan un papel crítico dentro del proceso de enculturación de los sujetos. De ahí que, durante la conferencia titulada "International Conference on Science Education in Developing Countries: From Theory to Practice", Ilevada a cabo en Israel en 1993, los investigadores Ilegaron al concenso de que el propósito fundamental de la educación en Ciencias debería ser la alfabetización científica de todos los ciudadanos. Puesto que esta ejerce una fuerte influencia en el momento de tomar una decisión informada en lo personal, local, nacional y global en cuanto a necesidades fundamentales de la sociedad (ej., alimento, energía, agua, conservación del ambiente, biotecnología, entre otras), la alfabetización científica es imperante.

Ahora bien, en el mundo se han producido diferentes conceptualizaciones del constructo de alfabetización científica, las cuales recogen los fines de esta educación de acuerdo con el sistema de creencias y valores de los diseñadores del currículo (Bybee y Ben-Zvi, 1998; Yore y Treagust, 2006). Sin embargo, de estas perspectivas subyace el presupuesto de que una persona científicamente alfabetizada es aquella que ha interiorizado un conocimiento de y sobre las ciencias, en conjunción con el desarrollado de las competencias lingüísticas de la oralidad, la lectura y la escritura. Así, la primera competencia le brinda la posibilidad al estudiante de comunicar dentro de un contexto social y divergente una representación intuitiva, la cual se convierte en el punto de partida para la coconstrucción de un modelo teórico con mayor nivel de elaboración. La segunda le permite seleccionar y evaluar la información presentada, desde una amplia oferta de fuentes documentales disponibles, con el fin de fundamentar la decisión a tomar. En cuanto a la tercera, le suministra la oportunidad de comunicar sus pensamientos y modelos mentales a través de recursos impresos o digitales a una audiencia particular con un propósito determinado, con el fin de persuadirla o convencerla (Holliday, Yore y Alvermann, 1994; Norris y Phillips, 1994). 
Por otro lado, los investigadores de educación en Química han formulado el interrogante sobre ¿cómo ayudar a los estudiantes a lograr una alfabetización científica? El tratar de dar solución a este conjunto de problemas los llevó a considerar que la enseñanza de un amplio abanico de contenidos de las ciencias apoyada solo en actividades experimentales, pero que no incrementa la alfabetización científica. Por consiguiente, resulta necesario en el aprendizaje de esta disciplina hacer énfasis en aspectos del desarrollo explícito de las competencias lingüísticas antes mencionadas dentro de un contexto sociocultural, lo cual le permite a los estudiantes comprender a profundidad las entidades y procesos que subyacen a los fenómenos químicos (Norris y Phillips, 1994).

En este sentido, el profesor debe diseñar y poner en funcionamiento actividades de aprendizaje direccionadas por el desarrollo de las competencias lingüísticas de la oralidad, la lectura y la escritura dentro de un contexto de indagación, con el propósito de mediar el logro de la alfabetización científica en la escuela. Naturalmente, estas competencias lingüísticas juegan y desempeñarán un papel vital en el logro de dicha meta en el marco de las actuales reformas curriculares de la educación en Química, considerando que estas pueden servir como estrategias conceptuales para ayudar al estudiante a analizar, interpretar y comunicar las ideas científicas a una audiencia particular con el fin de persuadirla (Holliday, Yore y Alvermann, 1994). Además, estas le permiten al estudiante comprometer sus modelos mentales durante los razonamientos complejos y la resolución de problemas, de manera análoga como los científicos las usan dentro de su comunidad de práctica.

Los anteriores presupuestos han comenzado a evidenciar que las competencias lingüísticas de la oralidad, la lectura y la escritura son estrategias potenciales para ayudar a desarrollar el pensamiento de los estudiantes y permitirles comprender a profundidad los fenómenos naturales (Durst y NeweII, 1989; Langer y Applebee, 1987; Rivard, 2004). No obstante, estas han sido usadas en el aula de
Química de manera separada, lo que ha provocado en los estudiantes una restricción para alcanzar una comprensión conceptual; caso contrario sucede cuando se logra combinar en el aula la oralidad, la lectura y la escritura con la intención de obtener los beneficios que aportan cada una de estas (Olson, 1994; Thaiss, 1988).

Si bien en las últimas décadas la educación en Química ha incrementado el interés por indagar sobre cómo las competencias lingüísticas de la oralidad, la lectura y la escritura ayudan a mediar el aprendizaje de los contenidos, las teorías y conclusiones provenientes de los diferentes estudios no han sido tomadas en consideración dentro de los programas de educación del profesor en formación y en ejercicio (Candela y Espinosa, 2016; Lemke, 1990). Quizás esta situación se da como consecuencia de que muchos formadores de profesores presentan la creencia que estas competencias son exclusivas de la educación en lenguaje, y las conciben como elementos accesorios y no constitutivos de las ciencias (Baker y Saul, 1994).

Finalmente, el propósito del presente estudio es comprender cómo el desarrollo explícito de las competencias lingüísticas de la oralidad, la lectura y la escritura en el aula de Química, desde una perspectiva sociocultural, media la comprensión del fenómeno del equilibrio químico. Tomando como punto de referencia los anteriores presupuestos se formula el siguiente interrogante, el cual direcciona la trayectoria a seguir en este estudio: ¿Cómo el desarrollo explícito de las competencias lingüísticas de la oralidad, la lectura y la escritura media la comprensión del equilibrio químico?

\section{Aspectos metodológicos de la investigación}

Este estudio se lleva a cabo por medio de una metodología cualitativa por estudio de casos, la cual se encuentra alineada con el problema y propósito que lo direcciona (Stake, 1998). Dicha decisión se fundamenta en la presunción de que esta perspectiva de indagación permite comprender a profundidad las acciones del profesor y los estudiantes, 
cuando el primero integra las competencias lingüísticas al aula de Química como una estrategia mediadora, para que los segundos alcancen la comprensión del fenómeno del equilibrio químico. Conviene subrayar que las unidades de análisis de este estudio son las interacciones dialógicas entre el profesor y los estudiantes, las cuales desempeñan un papel crítico para dar solución al problema bajo consideración. Ahora bien, para recoger y describir estas interacciones se emplean fuentes documentales, tales como: videos, notas de campo, entrevista y trabajos de los estudiantes.

Por otro lado, resulta importante precisar los criterios bajo los cuales se selecciona el caso de estudio (Stake, 1998). En primer lugar, se indaga por un profesor quien sea conocedor del lenguaje como un mediador de la enseñanza de las ciencias y que, además, se encuentre actualmente poniendo en marcha esta estrategia de enseñanza en el aula de Química. En segundo lugar, que se pueda acceder a él y a sus estudiantes. Finalmente, que los sujetos estudiados estén dispuestos a invertir tiempo y cognición para el desarrollo de la investigación.

Los anteriores criterios orientaron la búsqueda de un profesor que deseara participar en la investigación. Para ello, se revisó la base de datos de los maestros cooperados de una universidad pública de Cali, Valle del Cauca, logrando identificar 10 posibles casos. Posteriormente, se los contactó e invitó a una reunión en la que se socializó el propósito del estudio; además, se pidió permiso para observar una de sus clases de Química con la intención de verificar el primer criterio de selección. En este sentido, tres profesores tomaron la decisión de participar activamente en la investigación, pero, solo el maestro Carlos logró cumplir con el primer criterio de selección.

\section{Análisis de datos y codificación}

El análisis de los datos de este estudio se hizo a través de la teoría fundamentada de Strauss y Corbin (2002), la cual está estructurada por tres fases: descripción, ordenamiento conceptual y teorización.
Así, la primera se da por medio de la recolección sistemática de la información del caso; la segunda ofrece la posibilidad de inducir de los datos las categorías (tabla 1); y la última permite construir la teoría naturalística a partir de la selección de la categoría medular y el establecimiento de relaciones semánticas de esta con las otras categorías del estudio (codificación selectiva).

Tomando como referencia los anteriores enunciados que sustentan este análisis de naturaleza comparativa, se realizaron las siguientes tareas analíticas:

1. Organización rigurosa al conjunto de información recogida en las fuentes documentales de este estudio.

2. Lectura reflexiva al conjunto de documentos provenientes de las diferentes fuentes documentales, con el fin de encontrar pruebas que apoyen cómo la implementación de las competencias lingüísticas de la oralidad, la lectura y la escritura median el desarrollo de una profunda compresión del contenido del equilibrio químico. Para ello se realizó un proceso de codificación abierta tomando como referencia el problema y el marco conceptual de este estudio, permitiendo asignar a las respectivas unidades de registro los códigos que están alineados con las propiedades de estas.

3. Lectura y relectura al conjunto de códigos asociados a las unidades de registro para detectar patrones de ocurrencia, y de esta manera agruparlos de acuerdo con las similitudes encontradas de sus propiedades.

4. Fragmentación del cuerpo de conocimiento previamente codificado en unidades básicas de análisis con sentido independiente, con el propósito de reunir los códigos cuyo patrón de ocurrencia presentan similitud en sus propiedades. Esta acción analítica dio origen a las principales categorías de este estudio (tabla 1).

5. Adscripción de las unidades de análisis previamente codificadas (ej., párrafos con sentido independiente, eventos críticos de los videos), 
a cada una de las categorías inductivas con las que está en coherencia de acuerdo con sus propiedades (codificación axial).

Selección de la categoría medular o central (tabla 1), a la cual se vinculan de forma semántica las otras categorías. El propósito de dicha tarea analítica descansa en establecer relaciones de significado entre las respectivas unidades de análisis y, de esta manera, construir una teoría naturalistica que da solución al problema de investigación.

Tabla 1. Categorías inductivas producto del proceso de codificación.

\begin{tabular}{|c|}
\hline Categorías \\
\hline $\begin{array}{l}\text { 1. La oralidad, la lectura y la escritura en vínculo } \\
\text { con una gestión de aula sociocultural, factor cla- } \\
\text { ve en el aprendizaje del equilibrio químico. }\end{array}$ \\
\hline $\begin{array}{l}\text { 2. Integrando la lectura y la escritura en el aprendi- } \\
\text { zaje del equilibrio químico. }\end{array}$ \\
\hline $\begin{array}{l}\text { 3. El lenguaje verbal y escrito mediador de la com- } \\
\text { prensión del equilibrio químico. }\end{array}$ \\
\hline $\begin{array}{l}\text { 4. La instrucción explícita y fundamentada de la } \\
\text { oralidad, la lectura y la escritura, elemento funda- } \\
\text { mental para el desarrollo del discurso científico } \\
\text { en el aula de Química. }\end{array}$ \\
\hline
\end{tabular}

Fuente: Los autores.

\section{Resultados}

Por todo esto, resulta importante retratar el conjunto de acciones llevadas a cabo por el profesor y los estudiantes durante la comprensión del contenido del equilibrio químico. Para ello el análisis de los datos a través de la teoría fundamentada de Strauss y Corbin (1999) generó un conjunto de categorías, las cuales son desarrolladas teóricamente.

\section{La oralidad, la lectura y la escritura en vínculo con una gestión de aula sociocultural, factor cla- ve en el aprendizaje del equilibrio químico}

En esta clase de ambiente de aprendizaje la comprensión del fenómeno del equilibrio químico alcanzada por los estudiantes se construye de manera activa a través de procesos individuales y sociales dentro de una comunidad de aprendizaje. De ahí que no solo las actividades experimentales desempeñan un papel crítico, estas deben complementarse con el desarrollo de las competencias de la oralidad, la lectura y la escritura. Naturalmente, dichas competencias median la construcción, representación y comunicación de la comprensión del fenómeno químico dentro de un contexto sociocultural.

Conviene subrayar que en esta especie de escenario cada una de las contribuciones dadas por los estudiantes es respetada y consensuada, con el fin de construir un concenso que sintetiza las diversas perspectivas sobre el fenómeno químico provenientes de los pequeños grupos de discusión. En efecto, las competencias de la oralidad, la lectura y la escritura median la coconstrucción y negociación de los significados y formas de significar entre los aprendices. Además, estas constituyen el andamiaje del desarrollo de un lenguaje común que da identidad a la comunidad.

Definitivamente en este escenario áulico la oralidad, la lectura y la escritura, en vínculo con las actividades experimentales, motivan la indagación y la reflexión generando nuevos interrogantes e hipótesis, los cuales se traducen en investigación y comprensión. Situación que compromete a los estudiantes a suministrar retroalimentación el uno al otro con el propósito de comprender el fenómeno del equilibrio químico. Por tanto, esta orientación de enseñanza asume la construcción del conocimiento desde una perspectiva social y, a estas competencias lingüísticas herramientas de aprendizaje que median los procesos de negociación semántica, los cuales les permite a los aprendices ir reestructurando sus recursos lingüísticos.

La comunicación entre el profesor y los estudiantes en esta orientación de enseñanza sucede de forma horizontal; es decir, todos los integrantes, según sus antecedentes académicos y culturales, aportan elementos para dar solución a las tareas problemas que se están enfrentando. En este ambiente de apoyo y libre de amenaza, los estudiantes 
que aún no hayan alcanzado la comprensión del tema en cuestión pueden lograrla a través de la discusión con sus pares y profesor. Lo anterior se puede evidenciar por medio del siguiente testimonio y el texto realizado por un estudiante, el cual se encuentra en el enlace http://alejandravalencia914.wixsite.com/reacciones-quimicas/tema-1 (Trabajo, Alejandra).

En este año lectivo hemos visto muchos estrategias de enseñanza empleadas por el docente: la discusión en pequeños grupos, la discusión con toda la clase, la elaboración de textos escritos, la lectura de textos multimodales, la utilización de recursos tecnológicos digitales, el uso de prácticas de laboratorio, entre otras. Cada una de estas fueron experiencias maravillosas que nos facilitó una mayor comprensión de los temas de Química, utilizando estrategias innovadoras y temporales. Llevar nuestros argumentos con respecto al área de discusión en pequeños grupos de personas y posteriormente a toda la clase nos enfrentó a nuestro miedo al hablar en público, y agudizó nuestra postura y forma de argumentar frente a unos determinados ítems. Además de ponernos en una posición investigativa, en la que gracias a la redacción de textos escritos y la lectura de textos multimodales logramos la apropiación de dichos temas. Y junto con la constante utilización de tecnologías digitales y prácticas de laboratorio llegamos al punto donde nos encontramos con la forma de dar a evidenciar y experimentar aquellos conocimientos que adquirimos de forma textual, ahora sí, llevados a la práctica y de la mano con una explicación causal de las diversas problemáticas planteadas. (Entrevista, Claudia)

\section{Integrando la lectura y la escritura en el aprendi- zaje del equilibrio químico}

Las acciones Ilevadas a cabo por los miembros del colectivo áulico durante el desarrollo de la actividad denominada "la temperatura como factor que condiciona la velocidad de las reacciones bioquímicas", permiten evidenciar que esta propicia un escenario para asistir a los estudiantes en el desarrollo de estrategias de lectura y escritura comprensiva. Es decir, en esta aula de Química se generan los espacios donde el estudiante conoce e implementa la serie de estrategias de lectura comprensiva y escritura coherente sugeridas por los especialistas, con la intención de adquirir una independencia cognitiva.

De ahí que los estudiantes, por medio de la lectura, ponen a interaccionar el conocimiento previo, las experiencias simultáneas y la información accedida desde documentos impresos y otras fuentes, dentro de un contexto sociocultural centrado en la construcción de significados. Así, ellos procesan de manera interactiva la información transpuesta entre la percepción selectiva de la información proveniente del texto impreso o digital, las experiencias simultáneas (indagaciones concretas, discusiones, pensamientos) y su conocimiento personal (intertextualidad), y de esta forma logran diferenciar e integrar la idea de los factores que afectan la velocidad de una reacción química. El logro de esta meta se fundamenta por medio de la verificación, estructuración y reconceptualización de la relación establecida entre la información nueva y la que yace en la memoria permanente del sujeto.

El profesor Carlos estimula a los estudiantes a considerar el texto como un recurso para describir, interpretar, explicar, discutir, argumentar, criticar y coconstruir la comprensión del fenómeno de los factores que modifican la velocidad de una reacción química. Esta situación quizás los ayuda a desarrollar hábitos para investigar el significado del texto en un nivel más sutil, así como a evaluarlos con un rigor más crítico.

En tal sentido, el profesor Carlos cambió la enseñanza improductiva acerca de la lectura por una estrategia que contiene actividades directamente relacionadas con el texto. Estas exigen a los estudiantes leer párrafo por párrafo el cuerpo del documento con el que se están enfrentando, deteniéndose, reflexionando sobre lo que han leído $y$, posteriormente, parafraseando de manera tanto 
verbal como escrita las principales ideas representadas en cada uno de los párrafos. Naturalmente, dichas estrategias resultan ser productivas, siempre y cuando las actividades ofrezcan las condiciones a través de las cuales los estudiantes tomen conciencia del propósito específico de la lectura del texto, además del compromiso activo que deben tener a lo largo de dicha actividad. Estos presupuestos están alineados con las siguientes intervenciones:

Profesor: ¿En este párrafo el autor qué nos quiere comunicar?

Valentina: En ese párrafo el autor nos quiere decir que dependiendo del cambio de energía y de las reacciones, así mismo se clasifican estas en endotérmicas y exotérmicas.

Angie: En ese párrafo también se expresa los dos conceptos que hacen referencian a la forma como se clasifican las reacciones. Cuando esta reacción requiere de energía para llevarse a cabo se denomina una reacción endergónica; pero, si al contrario la reacción libera energía es considerada exergónica. Sin embargo, si la energía que participa en la reacción se manifiesta en forma de calor esas reacciones pasan a denominarse endotérmicas y exotérmicas respectivamente.

Profesor: Listo, ¿qué preguntas tienes, hija?

Karol: O sea, que el experimento que realizamos con el agua oxigenada y la papa se considera endergónica, porque para que el agua oxigenada reaccionara se necesitó la papa.

Profesor: ¿Qué opinan ustedes de lo que acaba de decir Karol? (Video, clase 3)

En definitiva, las habilidades de la escritura en conjunción con las de lectura median de manera sinérgica, la comprensión que los estudiantes desarrollan de contenidos tales como: teoría de las colisiones, teoría del complejo activado, reacciones reversibles y el principio de Le Chatelier. Desde luego, esta comprensión construida dentro de una comunidad de aprendizaje tiene la ventaja de ser significativa y transferible a otros contextos. Además, incorpora concepciones razonables acerca de las ideas representadas en el texto. Al igual como sucede en la lectura, la formación de conceptos es una consecuencia normal de la escritura constructiva.

En este sentido, los estudiantes en el aula de Química escriben acerca de contenidos como la teoría de las colisiones, la teoría del complejo activado y las reacciones reversibles; para ello, utilizan el conocimiento de las ciencias previamente aprendido, además de los procesos de razonamiento de esta disciplina. Desde luego, estos estudiantes han comenzado a desarrollar habilidades que incluyen la metacognición, la construcción y relación de ideas, la producción y revisión del texto, permitiéndoles tener un desempeño apropiado en la composición escrita a lo largo de los años de escolaridad en Química.

Por otra parte, cuando los estudiantes escriben sobre las ideas que estructuran el contenido del equilibrio químico, ellos procuran formularse unas metas de escritura de acuerdo con la audiencia específica a la que va dirigido el texto y, adicionalmente, planean cómo alcanzarlas. Además, construyen ideas pertinentes para sus metas y recuperan información específica de su memoria permanente, lógicamente intentando relacionar las proposiciones entre sí. Evidencia del desarrollo progresivo de la habilidad de escritura y la comprensión de las ideas en consideración, se pueden encontrar en el siguiente enlace: http://kevinb0914.wixsite.com/misitio-1/reacciones-y-teorias (Trabajo de estudiante).

\section{El lenguaje verbal y escrito mediador de la com- prensión del equilibrio químico}

El profesor Carlos, con el propósito de que sus estudiantes logren comprender el efecto de la concentración, la temperatura y la presencia de catalizadores sobre la velocidad de algunas reacciones químicas, los enfrenta con actividades experimentales, las cuales tienen como meta ayudar a desafiar las concepciones intuitivas. Además, él gestiona el aula en pequeños grupos de discusión y 
estructura interactiva con toda la clase, con el ánimo de que los aprendices establezcan diferentes perspectivas acerca de la comprensión del contenido y las sometan a debate, para posteriormente llegar a un consenso. También, les pide que la nueva comprensión sea traducida en una composición escrita que presente micro y macroestructura textual. En efecto, esta tarea sucede en un plano intrasubjetivo y metacognitivo, en el cual los aprendices, de manera individual o colectiva, representan por escrito la idea que inicialmente lograron interiorizar durante la transacción de significados.

Las acciones inteligentes del profesor Carlos durante la puesta en escena de la anterior actividad experimental deja ver la creencia de que el lenguaje verbal y el escrito son habilidades lingüísticas complementarias que median la comprensión del fenómeno de la cinética química. Así pues, concibe la habilidad de la oralidad como el recurso a través del cual los estudiantes expresan sus representaciones sobre un fenómeno químico. Además, esta se da dentro de una comunidad de aprendizaje, en la que ellos, a través de las relaciones interpersonales, negocian los significados y formas de significar, con el propósito de construir la solución más apropiada a una situación problemática. Desde luego, a lo largo de la negociación se clarifican y distribuyen ideas como producto de la formulación de interrogantes, hipótesis y explicaciones Ilevadas a cabo por los estudiantes dentro del aula de Química.

Por otro lado, las acciones del colectivo áulico durante esta clase de actividades de aprendizaje dejan ver que el lenguaje escrito es una estrategia que asiste a los estudiantes de forma individual o grupal cuando se encuentran reflexionando acerca de un fenómeno químico. De hecho, la composición textual en la clase del equilibrio químico es usada como un dispositivo de pensamiento que permite explorar, probar, reforzar y refinar el conocimiento existente, posibilitando el desarrollo de una nueva comprensión. De igual modo, en esta especie de aula se considera que la competencia de la escritura suministra la posibilidad para que los estudiantes organicen sus pensamientos sobre el equilibrio químico, a través de las relaciones semánticas que establece entre los términos con el fin de comunicar la comprensión alcanzada de forma consensuada.

En este sentido, Carlos argumenta que el lenguaje oral les permite a los estudiantes comunicar dentro de un contexto social y divergente una representación intuitiva, la cual se convierte en el punto de partida para la coconstrucción de un modelo teórico con mayor nivel de elaboración. Del mismo modo, cree que la habilidad de la escritura les brinda la posibilidad de relacionar las ideas previamente coconstruidas para comunicar la comprensión alcanzada, y de esta manera congelarla con el fin de que llegue a ser algo sobre lo que se pueda reflexionar. Naturalmente, dicha tarea de composición escrita se da dentro de un escenario de múltiples oportunidades que admiten reconsiderar las ideas clave, hallar y eliminar errores con el propósito de configurar la macro y la microestructura del texto, la cual representa alguna de las principales ideas que configuran el contenido del equilibrio químico. Estas asunciones se pueden evidenciar en la composición escrita realizada por tres estudiantes. Véase el siguiente vínculo:

http://alejandravalencia914.wixsite.com/reacciones-quimicas/tema-1 (Trabajo de estudiantes)

\section{La instrucción explícita y fundamentada de la ora- lidad, la lectura y la escritura, elemento funda- mental para el desarrollo del discurso científico en el aula de Química}

Sin duda, por medio de sus acciones a lo largo de la lección del equilibrio químico, el profesor Carlos deja ver la creencia que las artes del lenguaje son cruciales para la construcción del conocimiento de la Química; así pues, estas deben ser explícitamente enseñadas como parte de la instrucción de esta disciplina. En este sentido, el profesor genera el escenario donde los estudiantes tienen la posibilidad de aprender las reglas y significados de las prácticas del lenguaje de la Química, a través de las siguientes estrategias de enseñanza: análisis 
detallado de las características lingüísticas de algunos textos que representan un fenómeno químico, construcción conjunta de géneros orientada por el profesor, lectura y escritura centrada en la función y la forma de los textos.

El profesor de Química, durante el desarrollo de las actividades que configuran la lección del equilibrio químico, no solo se limita a que los estudiantes logren interiorizar el conocimiento sustantivo de esta disciplina, sino que les enseña aspectos semánticos y sintácticos de las artes del lenguaje (ej., macro y microestructura textual, manejo de conectores textuales, intertextualidad, función/forma de un texto, estrategias de lectura y escritura, entre otros), con el fin de que sean utilizados como una estrategia de aprendizaje. Igualmente, asume que las artes del lenguaje organizan y representan el pensamiento, por ello, los estudiantes necesitan ser introducidos explícitamente dentro de las prácticas del lenguaje de la Química con la intención de llegar a ser ciudadanos científicamente alfabetizados en el sentido fundamental y derivado. Así, la interiorización del conocimiento genérico de las habilidades de la oralidad, la lectura y la escritura suministran la posibilidad a los aprendices para desarrollar nuevas ideas y pensamientos sobre los fenómenos químicos.

Como resultado, el profesor Carlos se ha interesado cada vez más en precisar en el aula de Química las diferentes estrategias sugeridas por la literatura en educación en Ciencias acerca de las habilidades de la oralidad, la lectura y la escritura de esta disciplina. Naturalmente, el desarrollo consciente de estas habilidades lingüísticas les permite a los estudiantes darle sentido a las diferentes ideas que estructuran el contenido del equilibrio químico. De ahí que Carlos considere los siguientes presupuestos: a) los estudiantes aprenden de y sobre la Química hablando, leyendo y escribiendo; b) hablar acerca del contenido de un texto es especialmente productivo, con el fin de desarrollar la competencia de la argumentación apoyada en la evidencia; y c) de la misma forma como un estudiante aprende a hacer química al lado de un profesional competente, él también aprende a leer, escribir y hablar de química en compañía y direccionado por un profesional (profesor) que posee estas competencias, además, lo modela, critica, guía y apoya en el desarrollo del lenguaje de esta disciplina. Los anteriores enunciados se encuentran sustentados por los siguientes testimonios:

Profesor: Hoy vamos a analizar los escritos que ustedes les hicieron a sus padres, sobre el fenómeno de cinética química, reacción y velocidad de una reacción. Entonces va a leer Paola. Recuerden que sus escritos deben tener los tres tipos de párrafos: introducción, desarrollo y conclusión. Además, estos tienen que presentar una microestructura donde se articulan de manera coherente la idea principal con las respectivas ideas secundarias. No olvidar que los párrafos debidamente jerarquizados le suministran al texto una macroestructura textual, la cual le marca una dirección al potencial lector. Ahora bien, Paola, antes de que empieces a leer tu texto, me gustaría que nos cuentes cuál fue la estrategia que adoptaste para hacer la composición escrita.

Paola: Antes de comenzar a hacer el escrito realizamos un plan de escritura con el que ubicamos cuáles eran las ideas claves que mencionaríamos en el texto; luego, las organizamos en orden de generalidad donde cada una daría origen a un párrafo con coherencia y cohesión. Por ejemplo, en el párrafo de introducción se haría énfasis en el contenido a tratar y hacia quien va dirigido el texto, en el segundo párrafo comenzamos a explicar las reacciones bioquímicas, el siguiente párrafo abordó los factores que afectan la velocidad de una reacción química, que en este caso son la temperatura y las enzimas. De esta forma, continuamos desarrollando párrafos, los cuales representan el tema principal, que hace referencia a cómo incide la temperatura en las reacciones del cuerpo humano... (Video, diálogo profesor-estudiantes).

Profesor: ¿Qué opinan muchachos de la forma y función del texto realizado por Claudia?

Fernando: Pues la forma es buena, ya que, el texto presenta macroestructura en la que Claudia describe paso a paso los experimentos que permitieron comprender los factores que afectan la velocidad de una reacción química. 
Profesor: ¿Diego, qué opinas?

Diego: En mi opinión el texto está muy bien hecho, tiene las ideas claras, me gustó la forma como describió lo sucedido en los experimentos.

Profesor: ¿Ella solamente describió?

Diego: No, ella también explicó por qué y cómo sucedía la reacción química a nivel macroscópico y submicroscópico.

Profesor: Entonces, ¿qué tipo de género utilizó ella en su escrito?

Esperanza: Ella utilizó el género descriptivo.

Profesor: ¿En qué momento utilizó el género descriptivo?

Esperanza: Cuando describió el proceso del experimento, dado que, en este hizo un recuento muy detallado de lo sucedió en el fenómeno.

Profesor: ¿En qué momento utiliza un género explicativo?

Javier: Cuando explicó lo percibido a nivel macroscópico en términos moleculares.

Profesor: Entonces, ¿ ¿ustedes qué opinan de la estrategia utilizada por Claudia para la construcción del texto?

Idalia: Apropiada, ya que lo hizo con el propósito de que el lector pueda comprender lo sucedido en el experimento en cuanto a los factores que afectan la velocidad de las reacciones. También, el texto tiene una macroestructura y microestructura, esto le permite al lector poder entender lo que Claudia pretende comunicar.

Profesor: El texto tiene algunas restricciones, pero en términos generales está muy bien desarrollado. Como lo afirman ustedes, se mueve desde un mundo macroscópico a un mundo submicroscópico, desde un texto narrativo a un texto de género explicativo, además de eso goza de una macroestructura a nivel del texto y una microestructura con respecto al párrafo. (Video, diálogo profesor-estudiantes)

\section{Conclusiones}

Para empezar, se destaca que entre el desarrollo de las competencias lingüísticas de la oralidad, la lectura y las escritura, así como el proceso de aprendizaje del equilibrio químico, existe una relación simbiótica. De modo que el primero les ofrece la posibilidad a los estudiantes de construir una comprensión del fenómeno en cuestión, en tanto que el segundo, por medio de las actividades experimentales, permite promover el desarrollo de las competencias en cuestión. Por ejemplo, en este estudio de casos para que los estudiantes aprendieran el equilibrio químico emplearon las competencias de la lectura y la escritura con el fin de indagar y organizar información proveniente del texto escolar. En efecto, esta actividad se constituyó como un elemento esencial de las competencias lingüísticas dentro del aula de Química. En otro momento del desarroIlo de la lección, los aprendices son implicados en investigaciones y manipulación de materiales, situación que los ayuda comprender contenidos, tales como: factores que afectan la velocidad de una reacción química y principio de Le Châtelier; esta meta es alcanzada como consecuencia a las discusiones acerca del significado de las investigaciones, las cuales son asistidas por la oralidad, la lectura y la escritura que suministran herramientas para registrar las observaciones y las evidencias.

Si bien la comprensión del equilibrio químico resulta importante para el profesor Carlos, el desafío está en enseñar a los estudiantes a pensar, hablar, leer y escribir acerca de los fenómenos químicos. De ahí que se considere que el foco de hacer y aprender Química descansa en la observación e interacción con los objetos y eventos del mundo real; para ello, los estudiantes deben hacer predicciones sobre cómo funcionan estos, leer para indagar lo que otros han aprendido de dichos fenómenos y construir explicaciones escritas.

Este enfoque integrador de la enseñanza del equilibrio químico les permite a los estudiantes desarrollar la disposición a manejar y criticar lo que hablan, leen y escriben. Además de utilizar la comprensión alcanzada en otros contextos de la vida real. En efecto, esta perspectiva de la 
integración de dichas competencias lingüísticas al aula de Química vincula la construcción, organización y clarificación de los significados para ellos y otros miembros de la comunidad de aprendizaje, sirviendo a múltiples propósitos, en lugar de funcionar como un mecanismo que representa un conocimiento científico ya acabado.

Conviene subrayar que las actividades experimentales desempeñan un papel crítico en el logro de la alfabetización científica en el sentido fundamental y derivada de la propuesta realizada por el profesor Carlos. De hecho, estas proveen un escenario apropiado donde los estudiantes realizan observaciones reflexivas al fenómeno en cuestión y producen pruebas que los orientan a hallar modelos y evaluar sus predicciones, mientras establecen conexión entre los datos experimentales y la información fruto del proceso de indagación realizado a los textos. Esta tarea estimula la discusión colaborativa y la negociación de las explicaciones que median la comprensión del fenómeno químico, la cual es traducida en una composición textual dirigida a una audiencia determinada con un propósito particular.

Por otra parte, durante el desarrollo de las actividades de aprendizaje puestas en escena por el profesor Carlos, se evidencia que para lograr la alfabetización científica, en un sentido fundamental y derivado, es necesario una instrucción explícita tanto del contenido sustantivo y sintáctico de la Química como de las competencias lingüistas en consideración. De donde resulta que a lo largo de la enseñanza de los contenidos de la Química se debe representar de manera explícita las competencias para hablar, leer y escribir sobre las entidades y procesos que subyacen a los fenómenos químicos. Adicionalmente, para lograr esta meta se necesita que el conocimiento del profesor abarque no solo los elementos disciplinares y pedagógicos para la enseñanza de un contenido específico, sino también, aspectos referentes a cómo utilizar las competencias de la oralidad, la lectura y la escritura como una estrategia de pensamiento y aprendizaje.

\section{Referencias}

Baker, L.; Saul, W. (1994). Considering science and language arts connections: A study of teacher cognition. Journal of Research in Science Teaching, 31(9), 1023-1037. https://doi. org/10.1002/tea.3660310913

Bybee, R. W.; Ben-Zvi, N. (1998). Science curriculum: Transforming goals to practices. En: B. J. Fraser \& K. G. Tobin (Eds.), International handbook of science education (pp. 487498). Dordrecht, NLD: Kluwer. https://doi. org/10.1007/978-94-011-4940-2 28

Candela, B. F.; Espinosa, T. R. (2016). El lenguaje como una estrategia para el aprendizaje de los temas del currículo de las ciencias. Revista Bio-grafía. Escritos sobre la Biología, 9(17), 73-98. $\quad$ https://doi.org/10.17227/20271034. vol.9num.17bio-grafia73.88

Durst, R. K.; Newell, G. E. (1989). The uses of function: James Britton's category system and research on writing. Review of Educational Research, 59(4), 375-394. https://doi. org/10.3102/00346543059004375

Gabel, D. L.; Samuel, K. V.; Hunn, D. (1987). Understanding the particulate nature of matter. J. Chem. Educ, 64(8), 695-697. https://doi. org/10.1021/ed064p695

Glynn, S. M. (1991). Explaining science concepts: A teaching-with-analogies model. The psychology of learning science. En S. Glynn, R. Yeany y B. Britton (eds.), The psychology of learning science (pp. 219-240). Hillsdale, NJ: Erlbaum.

Glynn, S. M.; Muth, D. (1994). Reading and writing to learn science: Achieving scientific literacy. Journal of Research in Science Teaching, 31(9), 1057-1073. https://doi.org/10.1002/ tea.3660310915

Glynn, S. M.; Yeany, R. H.; Britton, B. K. (1991). A constructive view of learning science. En S. M. Glynn, R. H. Yeany y B. K. Britton (eds.), The psychology of learning science (pp. 3-19). Hillsdale, NJ: Erlbaum. 
Greenbowe, T. J.; Rudd II, J. A.; Hand, B. M. (2007). Using the science writing heuristic to improve students' understanding of general equilibrium. Journal of Chemical Education, 84(12), 2007 2011. https://doi.org/10.1021/ed084p2007

Halliday, M. A. K.; Martin, J. R. (2003). Writing science: Literacy and discursive power. Nueva York, EE. UU.: Taylor y Francis. https://doi. org/10.4324/9780203209936

Hodson, D. (2003). Teaching and learning science: Towards a personalized approach. Philadelphia: Open University Press.

Holliday, W. G.; Yore, L. D.; Alvermann, D. E. (1994). The reading-science learning-writing connection: Breakthroughs, barriers, and promises. Journal of Research in Science Teaching, 31(9), 877-893. https://doi.org/10.1002/tea.3660310905

Langer, J. A. \& Applebee, A. N. (1987). How writing shapes thinking (Research Report No. 22). Urbana, IL: National Council of Teachers of English.

Lemke, J. L. (1990). Talking Science: Language, Learning, and Values. Stamford, CT: Ablex.

Mayer, R. E. (1991). El futuro de la psicología cognitiva. Madrid: Alianza Editorial.

Norris, S. P.; Phillips, L. M. (1994) Interpreting pragmatic meaning when reading popular reports of science. Journal of Research in Science Teaching, 31(9), 947-967. https://doi.org/10.1002/ tea.3660310909

Olson, D. R. (1994). The world on paper: The conceptual and cognitive implications of writing and reading. Cambridge, UK: Cambridge University Press.

Pozo, J. I.; Gómez Crespo, M. A. (1998). Aprender y enseñar ciencia: del conocimiento cotidiano al conocimiento científico. Madrid: Ediciones Morata.

Prain, V.; Hand, B. (1996a). Writing for learning in the junior secondary science classroom: issues arising from a case study. International Journal of Science Education, 18(1), 117-128. https:// doi.org/10.1080/0950069960180110
Prain, V.; Hand, B. (1996b). Writing for learning in secondaryscience: Rethinking practices. Teaching and Teacher Education, 12(6), 609-626. https:// doi.org/10.1016/S0742-051X(96)00003-0

Rivard, L. P. (2004). Are language - based activities in science effective for all students, including low achievers? Science Education, 88(3), 420442. https://doi.org/10.1002/sce.10114

Rivard, L. P.; Straw, S. B. (2000). The effect of talk and writing on learning science: An exploratory study.ScienceEducation, 84(5), 566-593.https:// doi.org/10.1002/1098-237X(200009)84:5\%3C566::AID-SCE2\%3E3.0.CO;2-U

Spiegel, G. F.; Barufaldi, J. P. (1994). The effects of a combination of text structure awareness and graphic postorganizers on recall and retention of science knowledge. Journal of Research in Science Teaching, 31, 913-932. https://doi. org/10.1002/tea.3660310907

Stake, R. E. (1998). Investigación con estudio de casos. Madrid: Ediciones Morata.

Strauss, A.; Corbin, J. (2002). Bases de la investigación cualitativa. Técnicas y procedimientos para desarrollar la teoría fundamentada. Traducida por Eva Zimmerman. Medellín: Editorial Universidad de Antioquia, Facultad de enfermería.

Sutton, C. (1992). Words, science and learning. Londres: McGraw-Hill Education.

Thaiss, C. (1988). The future of writing across the curriculum. En: S. H. McLeod (Ed.), Strengthening programs for writing across the curriculum: New directions for teaching and learning (vol. 36, pp. 91-102). San Francisco: Jossey-Bass. https://doi.org/10.1002/ $\underline{\mathrm{t} .37219883612}$

Yore, L. D.; Treagust, D. F. (2006). Current realities and future possibilities: Language and science literacy - empowering research and informing instruction. International Journal of Science Education, 28(2-3), 291-314. https://doi. org/10.1080/09500690500336973 